\title{
Single Photons by Quenching the Vacuum
}

\author{
E. Sánchez-Burillo, ${ }^{1}$ L. Martín-Moreno, ${ }^{2}$ J. J. García-Ripoll, ${ }^{3}$ and D. Zueco ${ }^{2,4}$ \\ ${ }^{1}$ Max-Planck-Institut für Quantenoptik, D-85748 Garching, Germany \\ ${ }^{2}$ Instituto de Ciencia de Materiales de Aragón and Departamento de Física de la Materia Condensada, \\ CSIC-Universidad de Zaragoza, E-50009 Zaragoza, Spain \\ ${ }^{3}$ Instituto de Física Fundamental, IFF-CSIC, Calle Serrano 113b, Madrid E-28006 \\ ${ }^{4}$ Fundación ARAID, Paseo María Agustín 36, E-50004 Zaragoza, Spain
}

(Received 9 November 2018; published 1 July 2019)

\begin{abstract}
Heisenberg's uncertainty principle implies that the quantum vacuum is not empty but fluctuates. These fluctuations can be converted into radiation through nonadiabatic changes in the Hamiltonian. Here, we discuss how to control this vacuum radiation, engineering a single-photon emitter out of a two-level system (2LS) ultrastrongly coupled to a finite-band waveguide in a vacuum state. More precisely, we show the 2LS nonlinearity shapes the vacuum radiation into a non-Gaussian superposition of even and odd cat states. When the 2LS bare frequency lays within the band gaps, this emission can be well approximated by individual photons. This picture is confirmed by a characterization of the ground and bound states, and a study of the dynamics with matrix-product states and polaron Hamiltonian methods.
\end{abstract}

DOI: 10.1103/PhysRevLett.123.013601

Introduction.-Quantum fluctuations underly many physical phenomena, e.g., the Lamb shift [1] or a modification of the atomic decay. They also try to explain [2] the cosmological-constant problem [3]. Vacuum fluctuations can be converted into radiation by nonadiabatic changes of the electromagnetic environment [4], as in the dynamical Casimir [5-9], and Unruh effects [10], and the Hawking radiations $[11,12]$. All these processes are explained with free-field theories-quadratic Hamiltonians of harmonic oscillators - that result in Gaussian states [13]. To create vacuum radiation with nontrivial statistics we need nonlinearities, such as quantum emitters.

In this Letter, we study the conversion of vacuum fluctuations into single-photon radiation. We focus on waveguide QED [14], studying a two-level system (2LS) coupled to a finite-bandwidth environment of onedimensional bosonic modes. This low-dimensional realization of the spin-boson model [15] leads to enhanced light-matter interactions. We assume these interactions to be in the ultrastrong coupling regime, where the coupling is comparable to the excitation energy of the quantum emitter [16-22]. Under these conditions, we show how to convert vacuum fluctuations into individual photons. Our protocol consists of either abruptly switching on and off the lightmatter coupling constant, or moving the qubit gap in and out of the photonic band (we will show the equivalence of both protocols in the Supplemental Material [23]). We demonstrate that this process is mediated by photon bound states, which we characterize numerically and analytically. These states, once the emitter excitation energy approaches the band gap, allow the emission of individual photons without violating the parity constraints of the model.
Finally, we prove that the two-level system serves also as a detector of quantum fluctuations.

The main novelty of our work is that it presents the first example of single-photon Fock states emitted from vacuum. This is different from the emission that occurs when a 2LS is ultrastrongly coupled to a cavity [31,32]. There, the emission is formed by photon pairs due to parity conservation [33-37]. In the geometry considered in this Letter, the existence of qubit-field bound states allows triggering single photons from vacuum. Our theoretical proposal can be realized with superconducting circuits [38]. Flux or transmon qubits ultrastrongly coupled to a superconducting waveguide [20,21] should allow testing our results, enlarging the family of quantum-field theory ideas [7-9] that superconducting circuits can emulate-in particular, the manifestation of virtual photons, which is of current interest [39].

Model.-We study the spin-boson model, a continuum of bosonic modes coupled to a 2LS [40]

$$
H=\Delta \sigma^{+} \sigma^{-}+\sum_{k} \omega_{k} a_{k}^{\dagger} a_{k}+\sigma_{x} \sum_{k} g_{k}\left(a_{k}^{\dagger}+a_{k}\right) \text {. }
$$

The $\sigma^{ \pm}$are ladder operators of the 2LS and $\Delta$ is the excitation energy of the 2LS. The Pauli matrix $\sigma_{x}$ couples with strengths $g_{k}$ to the bosonic field operators $\left\{a_{k}^{\dagger}, a_{k}\right\}$ in momentum space. We consider a dispersion relation $\omega_{k}=$ $\Omega-2 J \cos k$ [Fig. 1(b)] with $N$ momenta $k \in[-\pi, \pi)$ and a band edge that allows us to control the vacuum emission. This $\omega_{k}$ results from a one-dimensional array of cavities with nearest-neighbors coupling [Fig. 1(a)] 


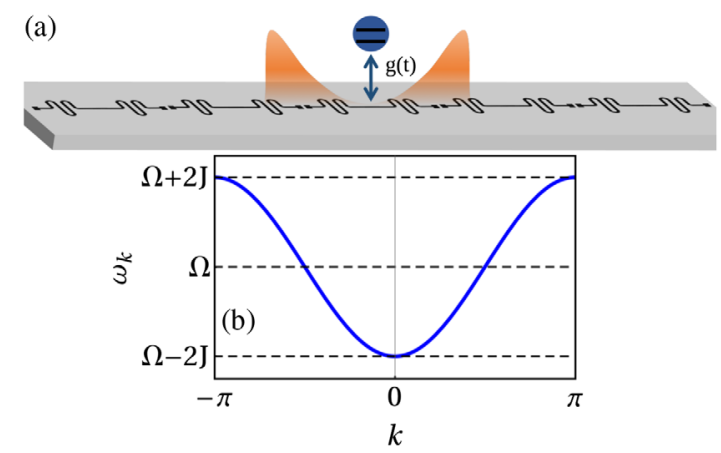

FIG. 1. (a) Sketch of the system. The 2LS-resonator interaction is $g(t)$. (b) Dispersion relation $\omega_{k}=\Omega-2 J \cos k$ of the model given by Eq. (2).

$$
H_{\text {array }}=\sum_{x=-N / 2}^{N / 2}\left[\Omega a_{x}^{\dagger} a_{x}-J\left(a_{x}^{\dagger} a_{x+1}+\text { H.c. }\right)\right] \text {, }
$$

with bosonic operators in positions $\left\{a_{x}, a_{x}^{\dagger}\right\}$, resonator frequency $\Omega$, and hopping $J$. This choice of photonic band is not essential, but favors the numerical simulation. The quantum emitter is coupled to a cavity at $x=0$, as in $H_{\text {coupling }}=g \sigma_{x}\left(a_{0}+a_{0}^{\dagger}\right)$, leading to $g_{k}=g / \sqrt{N}$ in Eq. (1).

If the coupling is sufficiently small, $g \ll \Delta$, the rotatingwave approximation (RWA) [41] allows us to replace the interaction term with $H_{\text {coupling }} \cong g\left(\sigma^{+} a_{0}+\right.$ H.c. $)$, which conserves the number of excitations. In this limit, the ground state has no excitations $|\mathrm{GS}\rangle_{\mathrm{RWA}}=|0 ; \mathbf{0}\rangle$ and is the product of the $2 \mathrm{LS}$ ground state $|0\rangle(|1\rangle$ is the excited state) and the zero-photon state of the waveguide $a_{k}|\mathbf{0}\rangle=0$. Therefore, under the RWA, the emitter is immune to the vacuum fluctuations of the bosonic field. However, the RWA fails in computing the actual vacuum properties $[42,43]$. Beyond the RWA, the ground state of (1) contains excitations: $\left\langle\mathrm{GS}\left|a_{x}^{\dagger} a_{x}\right| \mathrm{GS}\right\rangle \neq 0$, suggesting that the 2LS can convert fluctuations into radiated light. We investigate here the beyond-RWA vacuum emission of the spin-boson model (1).

Theoretical tools. - The spin-boson model is not solvable, except for a particular set of parameters and some limits, but matrix-product state (MPS) techniques can be used to obtain numerical results $[16,18,44]$, as explained in [23]. We contrast the numerical simulations with analytical approximations based on the polaron transformation [45-48]. This transformation is a disentangling operation $U_{p}$ that decouples the 2LS from the field

$$
U_{p}=\exp \left[-\sigma_{x} \sum\left(f_{k} a_{k}^{\dagger}-f_{k}^{*} a_{k}\right)\right]
$$

The parameters $f_{k}$ are obtained by minimizing the groundstate energy $E_{\mathrm{GS}}$ within the polaron ansatz for the ground state $|\mathrm{GS}\rangle=U_{p}|0 ; \mathbf{0}\rangle$, giving the equations

$$
f_{k}=\frac{g_{k}}{\Delta_{r}+\omega_{k}}, \quad \text { and } \quad \Delta_{r}=\Delta e^{-2 \sum_{k}\left|f_{k}\right|^{2}}
$$

The simplified Hamiltonian $H_{p}=U_{p}^{\dagger} H U_{p}$ reads

$$
\begin{aligned}
H_{p}= & \Delta_{r} \sigma^{+} \sigma^{-}+\sum_{k} \omega_{k} a_{k}^{\dagger} a_{k}-2 \Delta_{r}\left(\sigma^{+} \sum_{k} f_{k} a_{k}+\text { H.c. }\right) \\
& -2 \Delta_{r} \sigma_{z} \sum_{k, p} f_{k}^{*} f_{p} a_{k}^{\dagger} a_{p} \\
& +\frac{\Delta}{2}+\sum_{k}\left(\omega_{k}\left|f_{k}\right|^{2}-g_{k}^{*} f_{k}-f_{k}^{*} g_{k}\right)+\text { HOT. }
\end{aligned}
$$

Here, HOT stands for higher-order terms $\mathcal{O}\left(f^{3}\right)$. The transformed Hamiltonian conserves the number of excitations and can be treated analytically [48].

The renormalization of the $2 \mathrm{LS}$ energy $\Delta_{r}$ is a consequence of the coupling of a discrete quantum system to a continuum [40] (see [23]). According to the polaron picture, most correlations are captured by the unitary transformation of a product state $|\mathrm{GS}\rangle_{p}=|0 ; \mathbf{0}\rangle=U_{p}^{\dagger}|\mathrm{GS}\rangle$. Then, $U_{p}$ plays a similar role to the Bogoliubov transformations [49] used for finding the normal modes which account for the radiation in the Hawking, Unruh, or Casimir effects [4].

Spectrum of the spin-boson model.-The spectrum of the Hamiltonian (1) is essential to understand the dynamics of vacuum-induced photon emission. The photonic-band edge causes the appearance of photon bound states: localized excitations around the 2LS [16,50-54]. We classify those states according to their parity $\Pi=$ $\exp \left[i \pi\left(\sigma^{+} \sigma^{-}+\sum_{k} a_{k}^{\dagger} a_{k}\right)\right]$, which is a conserved quantity (1), $[\Pi, H]=\left[\Pi, H_{p}\right]=0$. More precisely, the ground state $|G S\rangle$ and the second bound state $\left|E_{2}\right\rangle$ are the first and second eigenstates with even parity $\Pi=+1$. The first bound state $\left|\Psi_{1}\right\rangle$ is the lowest eigenstate with odd parity $\Pi=-1 .\left|E_{1}\right\rangle$ and $\left|E_{2}\right\rangle$ have a well-defined number of particles in the RWA limit (1 and 2, respectively).

We compute these states using both MPS and the polaron Hamiltonian. Parity can be imposed during the MPS minimization of $H$; in the second case, we project the polaron Hamiltonian [Eq. (5)] onto spaces with fixed number of excitations, where it is numerically diagonalized. Figure 2(a) shows the energy of the ground state $E_{\mathrm{GS}}$ and of the first two bound states, $E_{1}$ and $E_{2}$, as a function of the coupling $g$. Note the excellent agreement between MPS (solid line) and the polaron Hamiltonian calculations (dots). Note also how the first bound state lays just below the onephoton band (gray band) $E_{1} \leq \epsilon_{k}(\mathrm{GS}) \equiv E_{\mathrm{GS}}+\omega_{k}$, just as in the RWA model [52-56]. The second excited bound state $E_{2}$ enters the band of propagating single photons. There may be other bound states, but the overlap with propagating photon bands of similar parity turn these bound states (which within the RWA would be perfectly localized) into resonances with a finite lifetime [16]. Further comparisons 

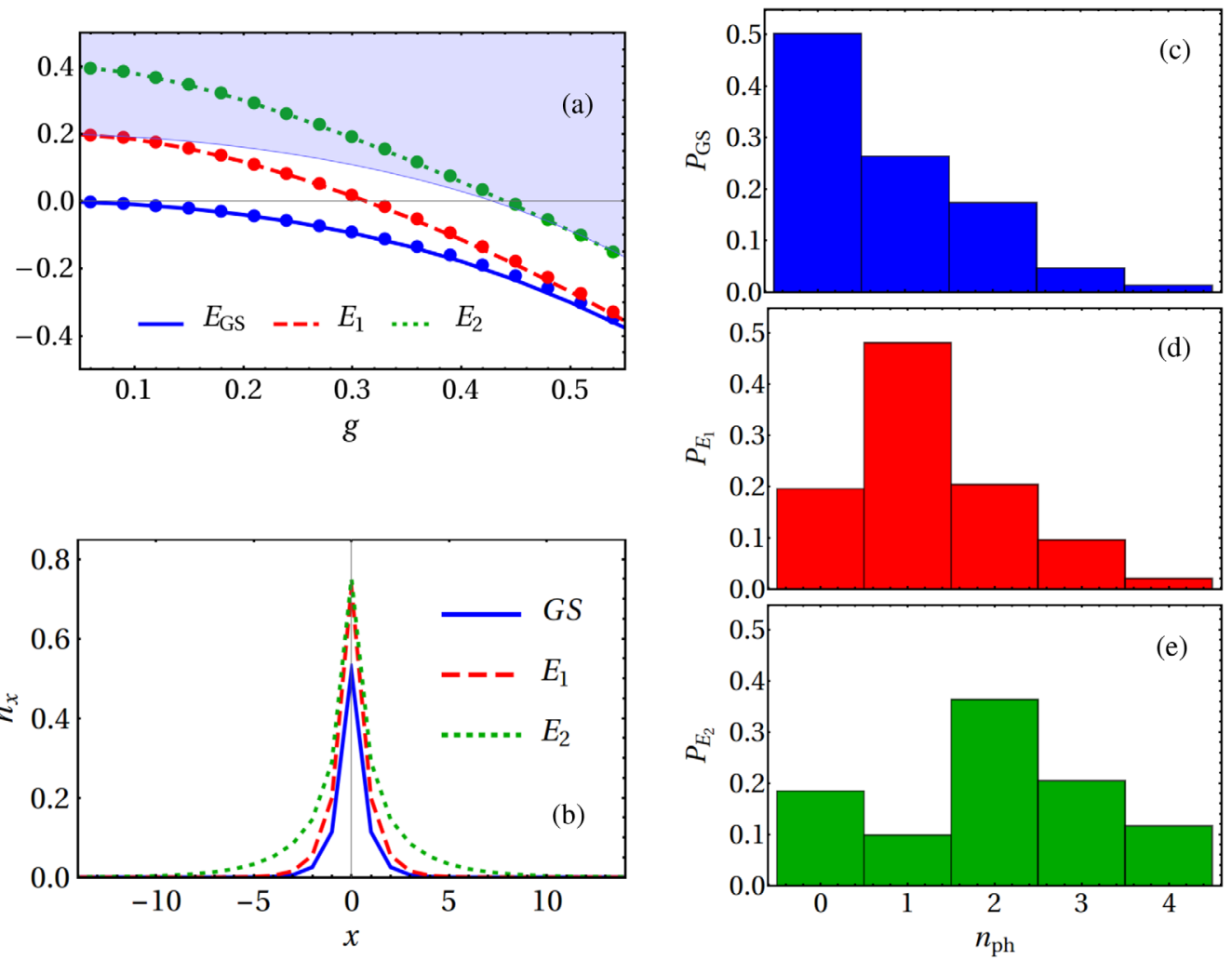

FIG. 2. Bound states. (a) Eigenenergies as a function of $g$ for $\Delta=0.3$. Continuous lines stand for the MPS simulations and the points for the polaron ansatz. (b) Bound states in position space for $g=0.5$ and $\Delta=0.3$. (c)-(e) Histograms with the weights in the $n_{\mathrm{ph}}$-photon sector for $|\mathrm{GS}\rangle,\left|E_{1}\right\rangle$, and $\left|E_{2}\right\rangle$. Same parameters as in panel (b). The parameters defining the photonic waveguide are $\Omega=1.0$ and $J=0.4$. The lattice length is $N=400$.

between results obtained using MPS and the polaron transformation are given in [23].

We have also analyzed the bound state MPS wave functions, $\left|E_{1}\right\rangle$ and $\left|E_{2}\right\rangle$. These states are localized around the 2LS, as seen in Fig. 2(b), which renders the number of photons in real space $\left\langle n_{x}\right\rangle=\left\langle a_{x}^{\dagger} a_{x}\right\rangle$. Interestingly, since the MPS produces wave functions in the original frame of reference-i.e., after applying $U_{p}$ onto the polaron stateswe find that these states are actual superpositions of different numbers of photons, as seen in Figs. 2(c)-2(e). The overall superposition preserves the parity of the state but, say, a bound state with two excitations can have a nonzero overlap with a single-photon component.

Emission by quenching the vacuum.-To convert vacuum excitations into emitted light, we consider a nonadiabatic protocol where the light-matter coupling strength is rapidly switched on and off. An alternative protocol, probably more amenable to experimental study, is to abruptly modify the qubit excitation energy $\Delta$ from a value that is strongly detuned from the photonic band $g /\left[\Delta(t<0)-\omega_{k}\right] \ll 1$ to a value $\Delta(t>0) \sim \omega_{k}$ while keeping a constant coupling $g$. Both methods are theoretically equivalent, since both ground states are the same up to an error $\exp \left[-g^{2} / \Delta\right]$ that can be made arbitrarily small [23].
In what follows, we analyze the coupling quench, which is simpler to describe both analytically and numerically, since the decoupled limit corresponds to $g=0$, while in the other case full decoupling occurs for infinite $\Delta$. We begin with an unexcited 2LS with $g(t<0)=0$ and switch on the coupling strength to a value $g(t=0)>0$ beyond the RWA regime. The $2 \mathrm{LS}$ immediately begins to emit light to accommodate its new ground state. The emitted photons form a wave packet that travels with speed $\max _{k}\left(\partial_{k} \omega_{k}\right)$. After some time the $2 \mathrm{LS}$ is no longer emitting and the wave packet leaves a cloud around the 2LS. We then suddenly switch off the coupling at $t=t_{\mathrm{off}}$ and a second vacuum emission takes place.

We simulate the dynamics described in the previous paragraph with MPS. The initial state is the trivial vacuum $|\Psi(t=0)\rangle=|0 ; \mathbf{0}\rangle$, which corresponds to the uncoupled case $g=0$, and $|\Psi(t)\rangle$ evolves under (1) with $g$ within the ultrastrong. In Fig. 3, we plot the photon number $n_{x}=$ $\left\langle a_{x}^{\dagger} a_{x}\right\rangle$ along the waveguide, as a function of time $t$ and position $x$. Note how all perturbations emerge from the 2LS position. We switch off the coupling once the traveling photons are far from the emitter. We choose $t_{\text {off }}=350 \tau$, being $\tau$ the spontaneous decay rate of the 2LS given by the Fermi's golden rule: $\tau \equiv J \sin \left(k_{0}\right) / g^{2}$, with $k_{0}$ such that 


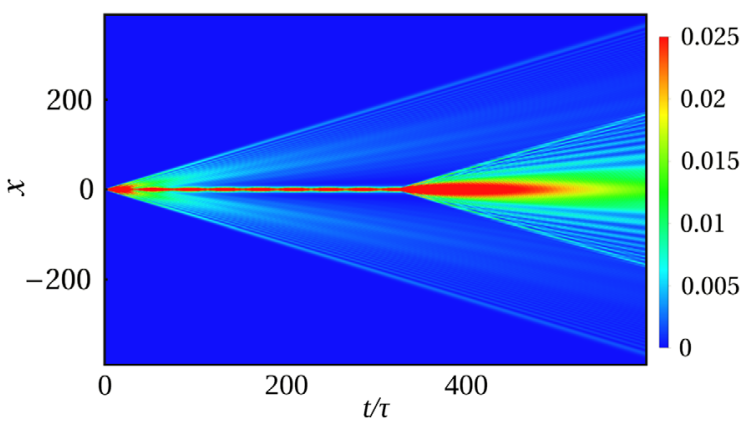

FIG. 3. Number of photons as a function of time and position for the quenching protocol: the initial state is the trivial vacuum $|\Psi(t=0)\rangle=|0 ; 0\rangle$ and the coupling is switched on at $t=0$. We switch $g$ off at $t_{\mathrm{off}} / \tau=350$. The system emits a wave packet at $t=0$. At $t=t_{\text {off }}$ it radiates again. $g=0.5$ after the initial quench and $\Delta=0.3$. The rest of parameters are as in Fig. 2 .

$\omega_{k_{0}}=\Delta$. At this point $g\left(t_{\text {off }}\right)=0$ and we witness the second photon emission event. Notice that photons propagate with different velocities because of the nonlinearity of the dispersion relation $\omega_{k}$ [see below Eq. (1)].

The whole process admits a simple description in the polaron picture. The state before the quench is

$$
|\Psi(t=0)\rangle_{p}=U_{p}^{\dagger}|0 ; \mathbf{0}\rangle=\frac{1}{\sqrt{2}}\left(\left|0 ; \boldsymbol{\alpha}_{+}\right\rangle+\left|1 ; \boldsymbol{\alpha}_{-}\right\rangle\right) .
$$

This is a superposition of even and odd cat states $\left|\alpha_{ \pm}\right\rangle \equiv e^{\sum\left(f_{k} a_{k}^{\dagger}-f_{k}^{*} a_{k}\right)}|\mathbf{0}\rangle \pm e^{-\sum\left(f_{k} a_{k}^{\dagger}-f_{k}^{*} a_{k}\right)}|\mathbf{0}\rangle$. In the limit of weak amplitudes, $\left|\alpha_{ \pm}\right\rangle$tend to one- and two-photon states, respectively [19], and the wave function can be written using bound and propagating states. Asymptotically in time, the state has the form

$$
\begin{aligned}
|\Psi(t)\rangle_{p}= & c_{0,0}(t)|0 ; \mathbf{0}\rangle+c_{0,2}(t)\left|E_{2}\right\rangle \\
& +c_{1,1}(t) A_{1 \gamma}^{\dagger}\left|E_{1}\right\rangle+c_{2,0}(t) A_{2 \gamma}^{\dagger}|0 ; \mathbf{0}\rangle+\cdots
\end{aligned}
$$

This wave function allows four possible outcomes: the system goes to (i) the ground state or (ii) to $\left|E_{2}\right\rangle$ with no emission; (iii) it relaxes to the first odd bound state $\left|E_{1}\right\rangle$ emitting a wave packet $A_{1 \gamma}^{\dagger}$ with one photon, or (iv) it relaxes to the ground state emitting two photons $A_{2 \gamma}^{\dagger}$. Note that when we write this wave function in the laboratory basis $|\Psi(t)\rangle=U_{p}|\Psi(t)\rangle_{p}$, the structure of the state is preserved, because the polaron transformation is local in space $\left[A_{1,2 \gamma}^{\dagger}, U_{p}\right]=0[23]$.

We have tested numerically that Eq. (7) captures the vacuum-triggered emission.

The simulations confirm that the system emits photons mainly in two channels: (i) one photon on the first excited odd bound state and (ii) two photons on the ground state, as predicted by Eq. (7). This is shown in Fig. 4(a), where we plot the number of photons $n_{x}$ at time $t / \tau=250$ and the
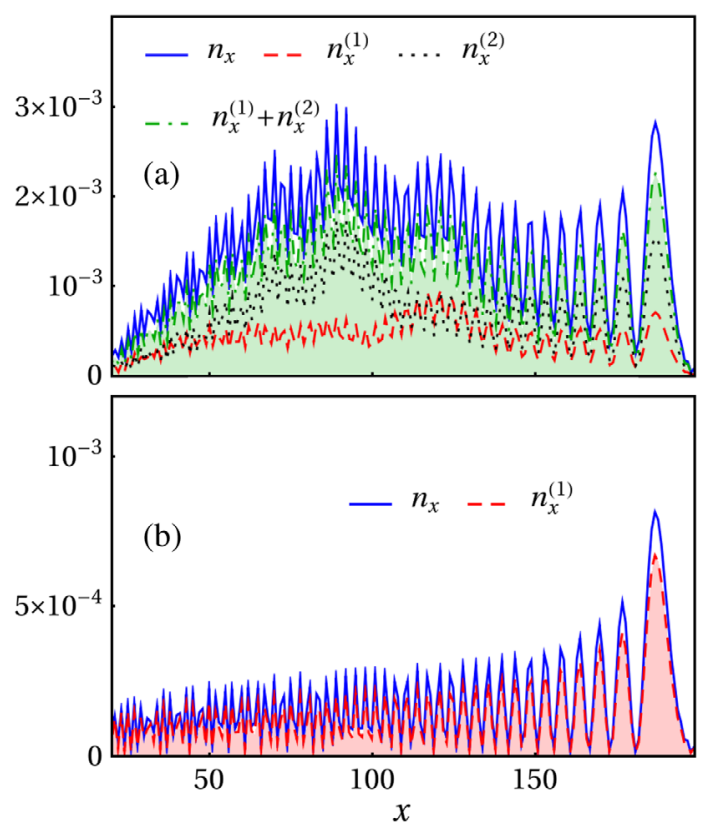

FIG. 4. (a) Number of photons at time $t / \tau=250$ after the quantum quench described in Fig. 3. We can approximate the field with one- and two-photon components. All the parameters are those of the previous figures (see Figs. 2 and 3). (b) Same as before, increasing the energy of the resonators $\Omega$ such that the band gap is now five times larger: $\Omega=1.8$ (keeping in mind that the band gap is $\Omega-2 J$ ). The system emits a single-photon packet.

single-photon $n_{x}^{(1)}=\left|\left\langle\Psi(t)\left|a_{x}^{\dagger}\right| E_{1}\right\rangle\right|^{2}$ and two-photon contributions $n_{x}^{(2)}=2 \sum_{x^{\prime}}\left|\left\langle\Psi(t)\left|a_{x}^{\dagger} a_{x^{\prime}}^{\dagger}\right| \mathrm{GS}\right\rangle\right|^{2}$. As seen, $n_{x}$ is well approximated by the sum of both wave packets $n_{x}^{(1)}+n_{x}^{(2)}$.

$|\Psi(t)\rangle_{p}$ also explains the second photon emission event. In this case, once we switch off the couplings, the bound states become unstable and decay, releasing their photonic components in the form of propagating photons. These come from the three first terms in Eq. (7). Two main features stand out. First, more power is radiated than in the first quench. This is because, in this second quench, excited bound states also radiate. Second, the radiated flying photons are slower. This is because the bound states are spectrally close to the photonic-band minimum, so radiation occurs mainly into slow photons. The distribution of this emitted light for each bound state matches the statistics in Figs. 2(c)-2(e).

The simulations prove that $|\Psi(t)\rangle_{p}$ also explains the 2LS dynamics [23].

We can control the vacuum-induced emission, for instance selecting the one-photon channel, by playing with the relative values of the band gap $\omega_{k=0}$ and the bound state energy $E_{1}-E_{\mathrm{GS}}$. The energies of the radiating states with one and two flying photons are $\epsilon_{k}\left(E_{1}\right)=E_{1}(g)+\omega_{k}$ and $\epsilon_{k_{1}, k_{2}}(\mathrm{GS})=E_{\mathrm{GS}}+\omega_{k_{1}}+\omega_{k_{2}}$, with respective minima 
$E_{1}(g)+\omega_{k=0}$ and $E_{\mathrm{GS}}+2 \omega_{k=0}$. If we place the emitter in the band gap $\omega_{k=0} \gg \Delta$, the energies $\epsilon_{k}\left(E_{1}\right)$ become closer to the 2LS resonance with respect to $\epsilon_{k_{1}, k_{2}}(\mathrm{GS})$, so the twophoton component is strongly suppressed $\left[\left|c_{2,0}\right| \simeq 0\right.$ in Eq. (7)]. The selectivity of this process is confirmed in Fig. 4(b), where the considered band gap is five times larger than in Fig. 4(a) and all other parameters are equal. The final state has a negligible overlap with $\left|E_{2}\right\rangle$ and the distribution of photons $P_{E_{n}}$ contains less than $1 \%$ of components with $n_{\mathrm{ph}} \geq 2$. The state before the second quench is faithfully reconstructed by just its single-photon component $A_{1 \gamma}^{\dagger}\left|E_{1}\right\rangle$, and as a result, the second emission is also well approximated by one photon.

Conclusions. - In this work we have studied the dynamics of vacuum fluctuations in ultrastrong waveguide-QED setups. More precisely, we have shown that the nonlinearity of a $2 \mathrm{LS}$, combined with a nonperturbative coupling to a bosonic field, can be used to create a vacuum-triggered single-photon emitter. In other words, we discuss the ultimate limit of quantum nonlinear optics as driven by vacuum fluctuations [57]. Our proposal is analogous in spirit to other quantum-field theory inspired proposals, such as the dynamical Casimir effect, which work with nonperturbative and nonadiabatic changes of the theory. In contrast to those experiments, we have shown a minimum setup which extracts single photons from vacuum, using bound states as mediators of these processes. It is important to remark that this whole study can be repeated using a resonator instead of a $2 \mathrm{LS}$. In this case, all of the features above disappear, as the emission has a Gaussian statistics that are not Fock states [58].

Our proposal and the conditions in this work can be realized in current circuit-QED devices with superconducting qubits that are ultrastrongly coupled to open transmission lines [20,21]. In this exciting platform, state-of-the-art measurement techniques would allow for a detailed reconstruction of the photon wave packets $[59,60]$.

We acknowledge the Spanish Ministerio de Ciencia, Innovación y Universidades within project MAT201788358-C3-1-R and FIS2015-70856-P and the Aragón Government project Q-MAD and CAM PRICYT Research Network QUITEMAD+ S2013/ICE-2801. EUQUANTERA project SUMO is also acknowledged. Eduardo Sánchez Burillo acknowledges the ERC Advanced Grant QUENOCOBA under the EU Horizon 2020 program (Grant Agreement No. 742102).

[1] W. E. Lamb and R. C. Retherford, Phys. Rev. 72, 241 (1947).

[2] V. Weisskopf and E. Wigner, Z. Phys. 63, 54 (1930).

[3] Q. Wang, Z. Zhu, and W. G. Unruh, Phys. Rev. D 95, 103504 (2017); F. D. Mazzitelli and L. G. Trombetta, Phys.
Rev. D 97, 068301 (2018); Q. Wang and W. G. Unruh, Phys. Rev. D 97, 068302 (2018).

[4] P. D. Nation, J. R. Johansson, M. P. Blencowe, and F. Nori, Rev. Mod. Phys. 84, 1 (2012).

[5] H. B. G. Casimir, Proc. K. Ned. Akad. Wet. 51, 793 (1948).

[6] S. K. Lamoreaux, Phys. Today 60, No. 2, 40 (2007).

[7] G. T. Moore, J. Math. Phys. (N.Y.) 11, 2679 (1970).

[8] P. Lähteenmäki, G. S. Paraoanu, J. Hassel, and P. J. Hakonen, Proc. Natl. Acad. Sci. U.S.A. 110, 4234 (2013).

[9] C. M. Wilson, G. Johansson, A. Pourkabirian, M. Simoen, J. R. Johansson, T. Duty, F. Nori, and P. Delsing, Nature (London) 479, 376 (2011).

[10] W. G. Unruh, Phys. Rev. D 14, 870 (1976).

[11] S. W. Hawking, Nature (London) 248, 30 (1974).

[12] S. W. Hawking, Commun. Math. Phys. 43, 199 (1975).

[13] G. Adesso, S. Ragy, and A. R. Lee, Open Syst. Inf. Dyn. 21, 1440001 (2014).

[14] D. Roy, C. Wilson, and O. Firstenberg, Rev. Mod. Phys. 89, 021001 (2017).

[15] U. Weiss, Quantum Dissipative Systems, 2nd ed. (World Scientific, Singapore, 2008).

[16] E. Sánchez-Burillo, D. Zueco, J. J. García-Ripoll, and L. Martín-Moreno, Phys. Rev. Lett. 113, 263604 (2014).

[17] B. Peropadre, J. Lindkvist, I. C. Hoi, C. M. Wilson, J. J. García-Ripoll, P. Delsing, and G. Johansson, New J. Phys. 15, 035009 (2013).

[18] E. Sánchez-Burillo, J. García-Ripoll, L. Martín-Moreno, and D. Zueco, Faraday Discuss. 178, 335 (2015).

[19] N. Gheeraert, S. Bera, and S. Florens, New J. Phys. 19, 023036 (2017).

[20] P. Forn-Díaz, J. J. García-Ripoll, B. Peropadre, J.-L. Orgiazzi, M. A. Yurtalan, R. Belyansky, C. M. Wilson, and A. Lupascu, Nat. Phys. 13, 39 (2017).

[21] J. Puertas Martinez, S. Leger, N. Gheeraert, R. Dassonneville, L. Planat, F. Foroughi, Y. Krupko, O. Buisson, C. Naud, W. Guichard, S. Florens, I. Snyman, and N. Roch, npj Quantum Inf. 5, 19 (2019).

[22] A. F. Kockum, A. Miranowicz, S. D. Liberato, S. Savasta, and F. Nori, Nat. Rev. Phys. 1, 19 (2019).

[23] See Supplemental Material at http://link.aps.org/ supplemental/10.1103/PhysRevLett.123.013601 for (i) an alternative protocol where we quench the gap of the twolevel atom, (ii) a brief introduction to matrix-product states, and (iii) further tests of the polaron transformation, which includes Refs. [24-30].

[24] J. Eisert, M. Cramer, and M. B. Plenio, Rev. Mod. Phys. 82, 277 (2010).

[25] G. Vidal, Phys. Rev. Lett. 91, 147902 (2003).

[26] G. Vidal, Phys. Rev. Lett. 93, 040502 (2004).

[27] F. Verstraete, J. J. García-Ripoll, and J. I. Cirac, Phys. Rev. Lett. 93, 207204 (2004).

[28] J. J. García-Ripoll, New J. Phys. 8, 305 (2006).

[29] F. Verstraete, V. Murg, and J. I. Cirac, Adv. Phys. 57, 143 (2008).

[30] M. Suzuki, J. Math. Phys. (N.Y.) 32, 400 (1991).

[31] T. Niemczyk, F. Deppe, H. Huebl, E. P. Menzel, F. Hocke, M. J. Schwarz, J. J. Garcia-Ripoll, D. Zueco, T. Hümmer, E. Solano, A. Marx, and R. Gross, Nat. Phys. 6, 772 (2010) 
[32] P. Forn-Díaz, J. Lisenfeld, D. Marcos, J. J. García-Ripoll, E. Solano, C. J. P. M. Harmans, and J. E. Mooij, Phys. Rev. Lett. 105, 237001 (2010).

[33] S. De Liberato, C. Ciuti, and I. Carusotto, Phys. Rev. Lett. 98, 103602 (2007).

[34] S. De Liberato, D. Gerace, I. Carusotto, and C. Ciuti, Phys. Rev. A 80, 053810 (2009).

[35] C. Ciuti, G. Bastard, and I. Carusotto, Phys. Rev. B 72, 115303 (2005).

[36] R. Stassi, A. Ridolfo, O. D. Stefano, M. J. Hartmann, and S. Savasta, Phys. Rev. Lett. 110, 243601 (2013).

[37] Q.-K. He, Z. An, H.-J. Song, and D. L. Zhou, arXiv:1810 $.04523 \mathrm{v} 1$.

[38] X. Gu, A. F. Kockum, A. Miranowicz, Y. xi Liu, and F. Nori, Phys. Rep. 718-719, 1 (2017).

[39] S. D. Liberato, Nat. Commun. 8, 1465 (2017).

[40] A. J. Leggett, S. Chakravarty, A. T. Dorsey, M. P. A. Fisher, A. Garg, and W. Zwerger, Rev. Mod. Phys. 59, 1 (1987).

[41] C. Cohen-Tannoudji, J. Dupont-Roc, and G. Grynberg, Atom-Photon Interactions: Basic Processes and Applications (Wiley-Interscience, New York, 1992).

[42] R. Loudon and S. M. Barnett, J. Phys. B 39, S555 (2006).

[43] P. R. Berman, R. W. Boyd, and P. W. Milonni, Phys. Rev. A 74, 053816 (2006).

[44] B. Peropadre, D. Zueco, D. Porras, and J. J. García-Ripoll, Phys. Rev. Lett. 111, 243602 (2013).

[45] R. Silbey and R. A. Harris, J. Chem. Phys. 80, 2615 (1984).

[46] S. Bera, A. Nazir, A. W. Chin, H. U. Baranger, and S. Florens, Phys. Rev. B 90, 075110 (2014).
[47] G. Díaz-Camacho, A. Bermudez, and J. J. García-Ripoll, Phys. Rev. A 93, 043843 (2016).

[48] T. Shi, Y. Chang, and J. J. García-Ripoll, Phys. Rev. Lett. 120, 153602 (2018).

[49] A. Altland and B. Simons, Condensed Matter Field Theory (Cambridge University Press, Cambridge, England, 2010).

[50] T. Shi and C. P. Sun, Phys. Rev. B 79, 205111 (2009).

[51] F. Lombardo, F. Ciccarello, and G. M. Palma, Phys. Rev. A 89, 053826 (2014).

[52] T. Shi, Y.-H. Wu, A. González-Tudela, and J. I. Cirac, Phys. Rev. X 6, 021027 (2016).

[53] G. Calajó, F. Ciccarello, D. Chang, and P. Rabl, Phys. Rev. A 93, 033833 (2016).

[54] E. Sánchez-Burillo, D. Zueco, L. Martín-Moreno, and J. J. García-Ripoll, Phys. Rev. A 96, 023831 (2017).

[55] P. Longo, P. Schmitteckert, and K. Busch, Phys. Rev. Lett. 104, 023602 (2010).

[56] P. Longo, P. Schmitteckert, and K. Busch, Phys. Rev. A 83, 063828 (2011).

[57] D. E. Chang, V. Vuletić, and M. D. Lukin, Nat. Photonics 8, 685 (2014).

[58] M. G. Genoni and M. G. A. Paris, Phys. Rev. A 82, 052341 (2010).

[59] E. P. Menzel, F. Deppe, M. Mariantoni, M. A. A. Caballero, A. Baust, T. Niemczyk, E. Hoffmann, A. Marx, E. Solano, and R. Gross, Phys. Rev. Lett. 105, 100401 (2010).

[60] C. Eichler, D. Bozyigit, C. Lang, L. Steffen, J. Fink, and A. Wallraff, Phys. Rev. Lett. 106, 220503 (2011). 